Z. Klin. Chem. Klin. Biochem.

11. Jg. 1973 , S. $308-310$

\title{
Probennehmergesteuerte Schaltautomatik zur Reduzierung des Reagenzienverbrauchs beim AutoAnalyzer
}

\author{
Von F. da Fonseca-Wollheim und G. Matgatter \\ Aus dem Institut fïr Klinische Chemie und Klinische Biocbemie (Direktor: Prof. Dr. H.-J. Dulce), \\ Klinikum Steglitz der Freien Universität Berlin
}

Eingegangen am 21. November 1972/9. März 1973)

Es wird ein Zusatzgerät für den Technicon-AutoAnalyzer beschrieben, das eine ökonomische Reagenziennutzung gewährleistet, indem es nach Beendigung jeder Probenserie selbsttätig die Reagenzienzufuhr unterbricht und auf Spüllösung umschaltet. Die Vorteile des Gerätes zeigen sich besonders bei Einsatz des AutoAnalyzers im ganztägigen on-line-Betrieb.

\section{Sampling controlled automatic switch, which decreases the consumption of reagent in the autoanalyzer}

An auxilliary apparatus for the Technicon Autoanalyzer is described, which permits the economic use of reagents. At the end of each sample series, the supply is automatically switched from reagent to washing solution. The advantages of this apparatus are most apparent with all day, on-line operation of the Autoanalyzer.

In meßbereitem Zustand verbrauchen kontinuierlich arbeitende Analysatoren vom Typ des AutoAnalyzers ständig Reagenzienlösung, unabhängig davon, ob Proben zur Messung zugeführt werden oder nicht. Theoretisch besteht zwar die Möglichkeit, zur Einsparung von Reagenz jeweils nach Beendigung einer Probenserie die Reagenzienschläuche in Spüllösung umzuhängen. In der Praxis hat sich aber gezeigt, daß beim 24-h-Betrieb eines Einkanal-AutoAnalyzers im klinisch-chemischen Laboratorium doch über weite Strecken Reagenzien vergeudet werden, da die für verschiedene andere Funktionen in Anspruch genommene diensthabende medizinisch-technische Assistentin dem Reagenzienverbrauch nicht genügend Aufmerksamkeit widmen kann.

Um die für Glucosebestimmungen mit dem AutoAnalyzer modifizierte Hexokinasemethode (1) wirtschaftlich ganztägig im on-line-Betrieb einsetzen zu können, haben wir ein Zusatzgerät für die Technicon-Apparatur gebaut, das - vom Probennehmer gesteuert - nach Bedarf zwischen Reagenz- und Spüllösung umschaltet. Außerdem besorgt diese Automatik auch noch das zeitgerechte An- und Abschalten des Schreibers, wodurch zusätzlicher Komfort bei der Bedienung des Analyzers gewonnen wird. Der Aufbau dieses Gerätes sowie seine Funktionsweise im Zusammenhang mit einem Einkanal-AutoAnalyzer werden hier beschrieben.

\section{Technischer Aufbau}

Der Reagenzpumpenschlauch ist durch eine Schlauchverbindung (Abb. 1, 4) an ein T-Stück angeschlossen, dessen zwei Arme mit Schlauchleitungen zu den beiden Vorratsgefäßen mit Reagenz bzw. Spüllösung führen (Abb. 1,1 und 1,2). Diese Schlauchleitungen (StandardTransportschlauch der $\mathrm{Fa}$. Technicon) sind durch ein

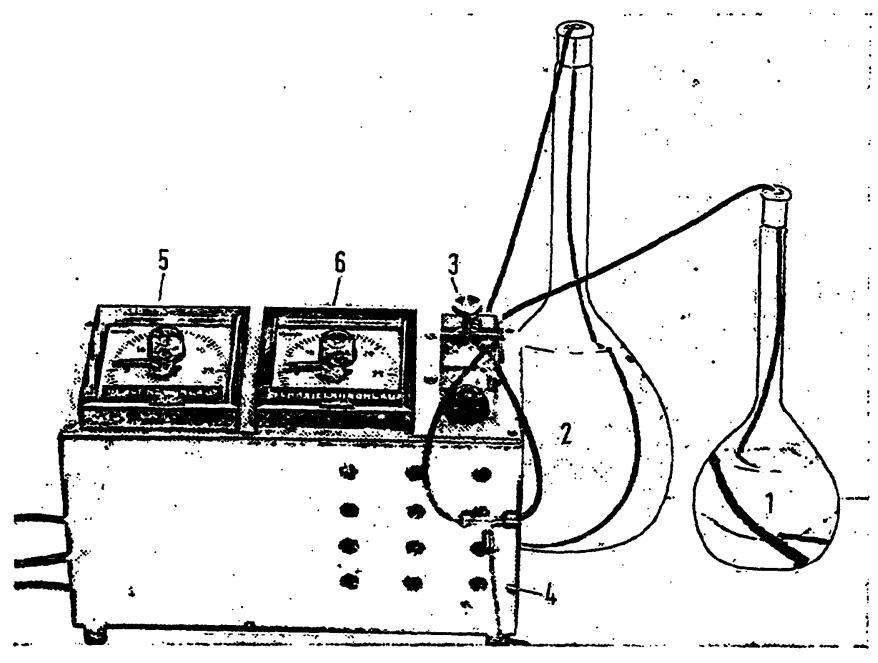

Abb. 1

Ansicht des Gerätes. Erläuterung siehe Text

Magnetventil (Abb. 1,3) geführt, das jeweils einen der beiden Schläuche abklemmt. Je nach Schaltung des Magnetventils wird also von der Schlauchpumpe Reagenz- oder Spüllösung angesaugt. Weitere wesentliche Bestandteile des Gerätes sind zwei Zeitrelais mit variabel einstellbarer Zeitvorgabe (Abb. 1,5 und 1,6), die für die zeitliche Koordination von Reagenzienzufuhr, Probennehmerfunktion und Registrierung am Schreiber benötigt werden (s. u.). Die beiden Relais sind mit dem Magnetventil in einer kompakten BauEinheit zusammengefaßt, die mit dem TechniconProbennehmer und dem Schreiber verkabelt ist. Die an Probennehmer und Schreiber erforderlichen Umbauten beinhalten nur unwesentliche Veränderungen in der Verdrahtung, wobei sich der ursprüngliche Zustand für manuelle Bedienung durch das Umlegen eines Schalters jederzeit wiederherstellen läßt (s. Schaltplan, Abb. 2). 


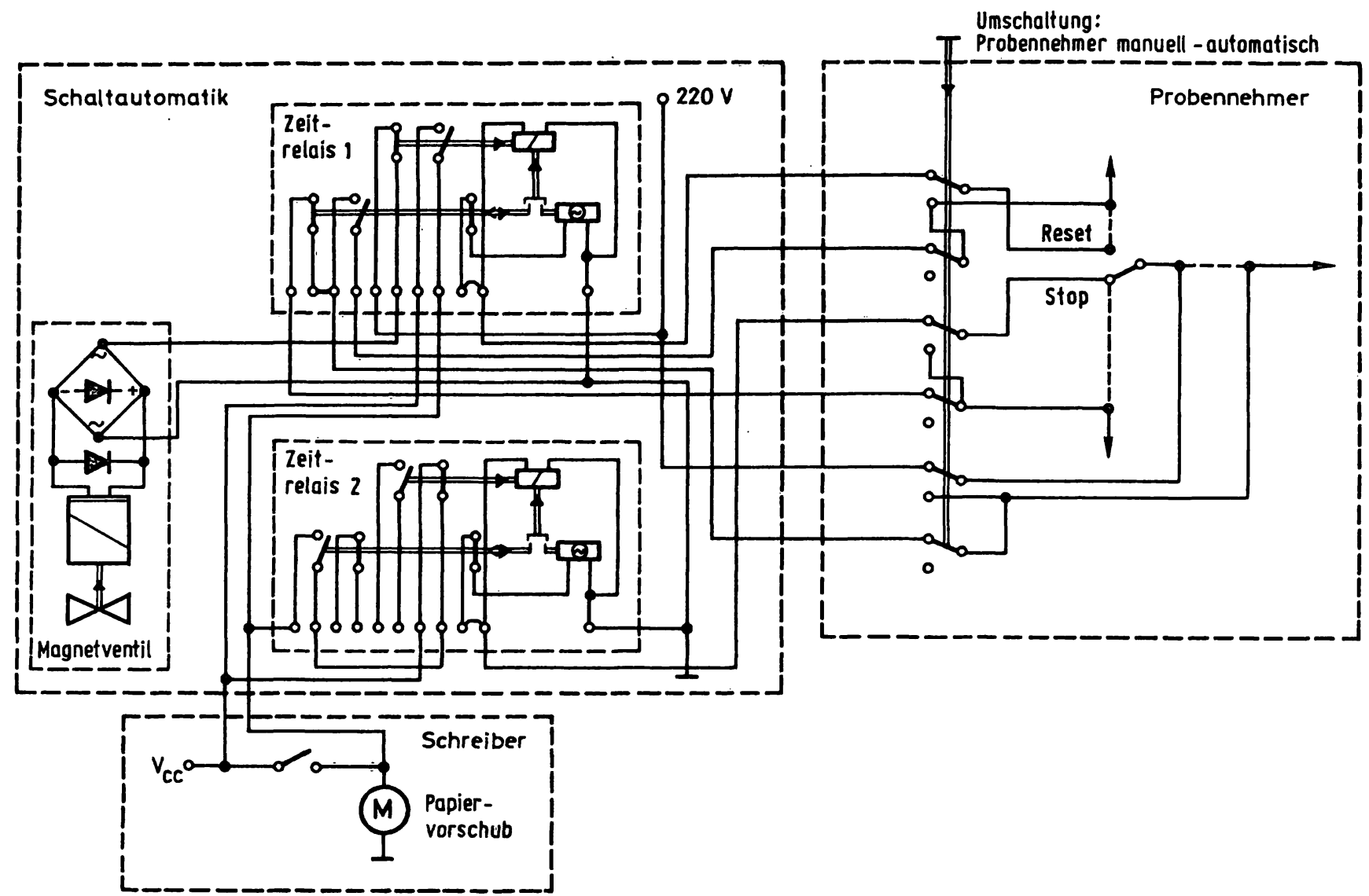

Abb. 2

Schaltplan der Anlage. -- -- ursprügliche Verkabelung des Probennehmers. Schalterstellung: Probennehmer automatisch, Probennehmer in Stopstellung, alle Zeitrelais abgelaufen

\section{Funktionsweise der Anlage}

Die Funktionsweise der Anlage ist aus dem Impulsdiagramm (Abb. 3) ersichtlich.

a) "Stand by-Zustand". Im Intervall zwischen den Probenserien verharrt der Probennehmer, durch den "Stopphaken" am Probenteller angehalten, in Ruhestellung. Die Schlauchpumpe läuft kontinuierlich und fördert Spüllösung durçh das System, da am Magnetventil der Reagenzienschlauch abgeklemmt und die Schlauchverbindung für Spüllösung freigegeben ist. Der Papiervorschub am Schreiber ruht.
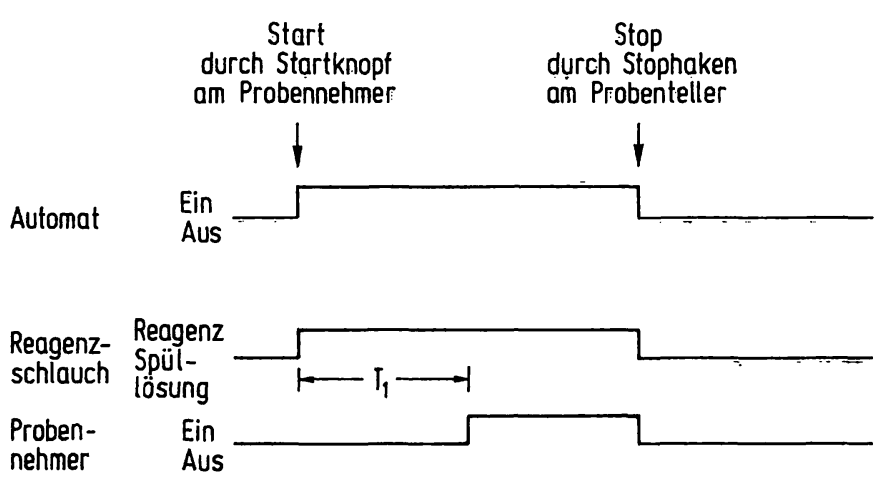

Schreiber

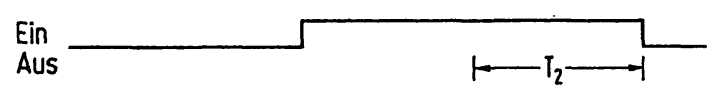

Abb. 3

Impulsdiagramm. $T_{1}$ Reagenzienvorlaufzeit; $T_{2}$ Schreibernachlaufzeit b) „Arbeitszyklus“. Sollen Proben analysiert werden, so wird durch Niederdrücken des „Reset“-Knopfes am Probennehmer der „Arbeitszyklus" gestartet. Dieses Umschalten bewirkt am Magnetventil Öffnung der Reagenzienzufuhr und Verschließen der Spüllösungszufuhr. Außerdem beginnt das eine Zeitrelais zu laufen. Erst wenn dieses nach der eingestellten Zeitspanne den Kontakt hergestellt hat, nimmt der Probennehmer seine Funktion auf (diese Zeitvorgabe für den Reagenzienstrom ist notwendig, damit bei Eintreffen des ersten Probensegments in der Inkubationseinheit dort bereits unverdünnte Reagenzlösung vorliegt). Über dasselbe Zeitrelais wird gleichzeitig die Registrierung am Schreiber in Gang gesetzt, so daß nun alle Teile der Technicon-Apparatur ihren normalen Betriebszustand erreicht haben.

Wenn aus dem letzten Probengefäß der Serie Material entnommen ist, wird der Probennehmer in üblicher Weise gestoppt, indem der in die entsprechende Position des Probentellers eingehängte „Stopphaken“ einen Mikroschalter berührt. Die Betätigung des Mikroschalters löst gleichzeitig am Magnetventil wieder Umschaltung auf Spüllösung aus und setzt das zweite Zeitrelais in Gang. Bis zum Ablauf der vorgewählten Zeit läuft die Registrierung am Schreiber weiter („Schreibernachlauf“, s. Abb. 3). Die Zeiteinstellung an diesem Relais wird so gewählt, daß der Schreiber erst nach Registrierung des letzten Probenpeaks und 
eines kurzen Abschnitts der darauf folgenden Basislinie abgeschaltet wird. Am Ende des Arbeitszyklus befindet sich die Anlage somit wieder im stand-byZustand.

\section{Diskussion}

Der hohe Reagenzienverbrauch bei Dauerbetrieb des AutoAnalyzers ist der Grund dafür, $\mathrm{da} B$ in vielen Kliniklaboratorien beispielsweise für die Blutglucosebestimmung noch immer Methoden mit billigen Reagenzien angewendet werden, die wegen ihrer mangelhaften Spezifität als überholt angesehen werden müssen. Wo anspruchsvollere enzymatische Methoden mit hochwertigen Reagenzien am AutoAnalyzer in Gebrauch sind, wird zur Einsparung von Reagenzienkosten im Spät- und Nachtdienst oft auf manuelle Ersatzmethoden ausgewichen. Diese organisatorische Lösung ist aber in verschiedener Hinsicht (Vergleichbarkeit der Resultate, Dokumentation der Meßwerte, Arbeitsbelastung für die diensthabende medizinischtechnische Assistentin) ebenfalls unbefriedigend.

\section{$\vdots i$}

\section{Literatur}

1. DA Fonseca-Wollhetm, F. (1973), diese Z. 11, 24-30.
Das hier beschriebene Zusatzgerät beschränkt den Reagenzienverbrauch des AutoAnalyzers auf die Zeit des effektiven Bedarfs und ermöglicht damit auch die Anwendung hochspezifischer enzymatischer Methoden mit teuren Reagenzien im 24-h-Dauerbetrieb. Wir haben in unserem Routinelaboratorium bei der Einführung des Gerätes am Glucosearbeitsplatz die Erfahrung gemacht, daß fast $50 \%$ der Reagenzienkosten gespart werden. Demgegenüber fäll't der bei ununterbrochenem Laufen der Schlauchpumpe gegebene etwas höhere Verschleiß an Pumpenschläuchen nur wenig ins Gewicht. Ein Wechsel der Pumpenschlauchgarnitur ist nach unseren Erfahrungen mit der Technicon-Proportionierpumpe II nach 100-150 Betriebsstunden erforderlich. Zusätzliche Vorteile bei der gewählten Betriebsweise sind die weitere Bedienungsvereinfachung des AutoAnalyzers und die kontinuierliche Spülung des Systems zwischen den Probenserien mit dest. Wasser.

\section{Danksagung}

Den Mitarbeitern der Technischen Abteilung unseres Hauses, insbesondere Herrn H. SCHWARZ, möchten wir für ihre bereitwillige Kooperation beim Bau des Gerätes danken.
Dipl. Ing. G. Maigatter

Institut für Klinișche Chemie und Klinische Biochemie Klinikum Steglitz der FU Berlin

1 Berlin 45

Hindenburgdamm 30 